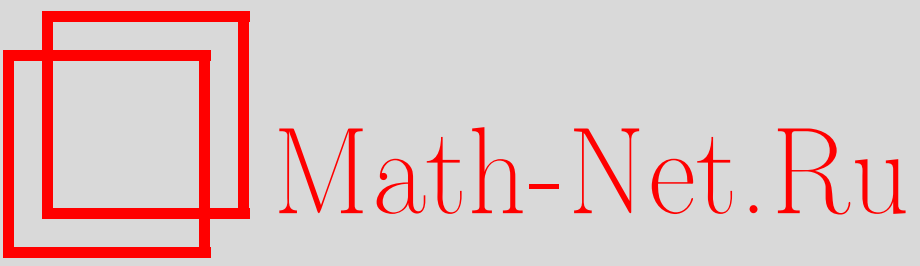

И. Ю. Попов, Д. А. Зубок, Два физических приложения оператора Лапласа, возмущенного на множестве нулевой меры, ТMФ, 1999, том 119, номер 2, 295307

DOI: https://doi.org/10.4213/tmf740

Использование Общероссийского математического портала Math-Net.Ru подразумевает, что вы прочитали и согласны с пользовательским соглашением http://www . mathnet.ru/rus/agreement

Параметры загрузки:

IP: 18.234 .197 .8

26 апреля 2023 г., $11: 23: 33$ 
ТЕОРЕТИЧЕСКАЯ

И МАТЕМАТИЧЕСКАЯ

ФИЗИКА

Том 119, № 2

май, 1999

(C) 1999 г.

Д. А. Зубок* , И. Ю. Попов

\section{ДВА ФИЗИЧЕСКИХ ПРИЛОЖЕНИЯ ОПЕРАТОРА ЛАПЛАСА, ВОЗМУШЕННОГО НА МНОЖЕСТВЕ НУЛЕВОЙ МЕРЫ}

Предлагаются два физических применения оператора Лапласа, возмушенного на множестве нулевой меры. Подход основан на теории самосопряженных расширений симметрических операторов в пространствах Гильберта и Крейна. Первое применение - явнорешаемая модель рассеяния плоской волны возмущенным тонким цилиндром. Описываются "нелокальные" расширения. Показано, что параметры модели могут быть выбраны так, что модельное решение дает приближение соответствующего "реального" решения. Второй пример есть приложение к описанию временной эволюции одномерной квазичаплыгинской среды, которая с использованием преобразования годографа сводится к некорректно поставленной задаче для оператора Лапласа, возмущенного на множестве коразмерности 2 в $\mathbf{R}^{3}$. Получены условия стабильности /нестабильности.

\section{1. ВВЕДЕНИЕ}

Задача о рассеянии волны тонким препятствием привлекает большое внимание. Развиваются различные асимптотические подходы для исследования рассеяния цилиндрическими и коническими поверхностями и их возмушениями [1-3]. Для изучения этой задачи мы предлагаем модель теории расширений операторов. Подход аналогичен хорошо известному методу потенциалов нулевого радиуса в квантовой механике, идея которого была предложена Ферми [4]. Березин и Фаддеев показали [5], что с математической точки зрения введение потенциала нулевого радиуса заключается в построении самосопряженного расширения некоторого симметрического оператора. Подход был обобщен, и в результате возникло много приложений в различных областях физики (см. книгу [6] и обзор [7]).

Мы предлагаем оператор Лапласа, возмушенный на линии в $\mathbf{R}^{3}$, как модель для задачи рассеяния, о которой идет речь. Проведено сравнение с асимптотикой (по диаметру цилиндра) "реального" решения. Показано, что параметры нелокального расширения могут быть выбраны таким образом, чтобы модельное решение совпадало с первым членом асимптотики "реального" решения.

* Кафедра высшей математики Ленинградского института точной механики и оптики, Санкт-Петербург, Россия 
Во второй части статьи описывается поведение так назьваемой квазигазовой нестабильной среды. Задача интересна своими физическими приложениями (в физике плазмы, в проблеме возникновения галактики; см., например, [8-10]). Она является сушественно нелинейной, но в некоторых случаях может быть сведена к линейной с использованием преобразования годографа. Мы будем иметь дело только с такой ситуацией. Это будет некорректно поставленная задача для оператора Лапласа, возмушенного на множестве нулевой меры. Корректное описание соответствуюшего оператора дается в рамках операторной теории расширений. Опишем коротко постановку задачи.

Рассмотрим временную эволюцию 1D квазичаплыгинской среды, которая описывается следуюшими уравнениями [11]:

$$
\rho_{* t}^{\prime}+\left(\rho_{*} v\right)_{x}^{\prime}=0, \quad v_{t}^{\prime}+v v_{x}^{\prime}=c_{0}^{2} m\left(\rho_{*}^{1 / m}\right)_{x}^{\prime} .
$$

Здесь $p_{0}, \rho_{0}$ - невозмушенные давление и плотность, $c_{0}^{2}=p_{0} / \rho_{0}>0, \rho_{*}=\rho / \rho_{0}, v=$ $v(x, t)$ - скорость, $m$ - некоторая константа (для газа Чапльгина $m=-1 / 2$ ).

ЗАмечАниЕ. Для реального газа имеем $\partial p / \partial \rho>0$, но для квазичаплыгинского газа связь другая: $\partial p / \partial \rho<0$.

Уравнения (1) являются нелинейными, но можно получить линейные уравнения, используя преобразование годографа. Процедура такова. Отышем обратные функции $t=t\left(\rho_{*}, v\right), x=x\left(\rho_{*}, v\right)$ вместо $\rho_{*}(x, t)$ и $v(x, t)$. Тогда

$$
v_{t}^{\prime}=\frac{x_{\rho_{*}}^{\prime}}{J}, \quad \rho_{* t}^{\prime}=-\frac{x_{v}^{\prime}}{J}, \quad v_{x}^{\prime}=-\frac{t_{\rho_{*}}^{\prime}}{J}, \quad \rho_{* x}^{\prime}=\frac{x_{v}^{\prime}}{J},
$$

где $J=x_{\rho_{*}}^{\prime} t_{v}^{\prime}-x_{v}^{\prime} t_{\rho_{*}}^{\prime}$ (якобиан). После подстановки (2) в (1) приходим к линейным уравнениям

$$
x_{v}^{\prime}=v t_{v}^{\prime}-\rho_{*} t_{\rho_{*}}^{\prime}, \quad x_{\rho_{*}}^{\prime}=v t_{\rho_{*}}^{\prime}+c_{0}^{2} t_{v}^{\prime} \rho_{*}^{-1+1 / m} .
$$

Условие совместности уравнений (3) есть следующее уравнение для функции $t\left(\rho_{*}, v\right)$ :

$$
\rho_{*}^{-1 / m}\left(\rho_{*}^{2} t_{\rho_{*}}^{\prime}\right)_{\rho_{*}}^{\prime}+c_{0}^{2} t_{v v}^{\prime \prime}=0
$$

Введем переменные

$$
r=\rho_{*}^{1 /(2 m)}, \quad z=\frac{v}{2 m c_{0}} .
$$

Тогда (4) принимает форму

$$
\widetilde{\Delta} t=t_{z z}^{\prime \prime}+t_{r r}^{\prime \prime}=-(1+2 m) r^{-1} t_{r}^{\prime}
$$

Для газа Чапльгина с $m=-1 / 2$ получаем уравнение Лапласа $\widetilde{\Delta}(t, z)=0$. При $m \neq$ $-1 / 2$ мы вводим функцию $\psi(r, z)=r^{m} t$, для которой (6) переходит в уравнение

$$
\psi_{z z}^{\prime \prime}+\psi_{r r}^{\prime \prime}+r^{-1} \psi_{r}^{\prime}-m^{2} r^{-2} \psi=0 .
$$

Добавим (формально) третью переменную $\phi$ (угол) к $r, z$ и рассмотрим $r, \phi, z$ как цилиндрические координаты. Тогда для вспомогательной функции $\Phi(r, \phi, z)=\psi(r, z) \times$ $\cos m \phi$ имеем трехмерное уравнение Лапласа

$$
\Delta \Phi=0
$$

вместо (7). Ниже мы будем иметь дело с некоторыми частными его решениями.

Статья состоит из двух частей. Первая часть (разделы 2-6) посвящена рассеянию на цилиндре, вторая (раздел 7) описывает модель квазичапльгинской среды. 


\section{2. МОДЕЛЬ РАССЕЯНИЯ ВОЛНЫ НА ЦИЛИНДРЕ}

Мы рассмотрим рассеяние на линии как модель для рассеяния на тонком цилиндре. Пусть $L$ - ось $O Z$ цилиндрической системы координат. В качестве исходного возьмем оператор Лапласа $A_{L}$, суженный на множество функций, обрашаюшихся в нуль на линии $L$. Оператор является симметричным с бесконечными индексами дефекта. Для того чтобы построить самосопряженное расширение, необходимо рассмотреть область определения сопряженного оператора. Нетрудно видеть, что

$$
D\left(A_{L}^{*}\right)=D\left(A_{L}^{\mathrm{F}}\right)+N_{\lambda_{0}},
$$

$A_{L}^{\mathrm{F}}$ есть расширение $A_{L}$ по Фридрихсу, $N_{\lambda_{0}}-$ ядро оператора $A_{L}^{*}-\lambda_{0}, \lambda_{0}=k_{0}^{2}<0$ есть некоторая регулярная точка оператора. Опишем множество $N_{\lambda_{0}}$. Видим, что $v$ $\left(v \in N_{\lambda_{0}}\right)$ удовлетворяют уравнению

$$
\left(\Delta+k_{0}^{2}\right) v(x)=\int_{-\infty}^{\infty} \delta((r, \phi, z)-(0, \phi, \tilde{z})) \alpha(\tilde{z}) d \tilde{z}
$$

здесь $\alpha(z)$ - некоторая функция на $\mathbf{R}$. Используя одномерное преобразование Фурье $F$ $(\tilde{v}=F v)$, получаем

$$
\begin{aligned}
\tilde{v}(r, \phi, \xi) & =\frac{i}{4} \int_{0}^{\infty} d r^{\prime} \int_{-\infty}^{\infty} d z H_{0}^{(1)}\left(\sqrt{k_{0}^{2}-\xi^{2}}\left(r-r^{\prime}\right)\right) \delta\left(r^{\prime}\right) \delta(\xi-z) \alpha(z)= \\
& =\frac{i}{4} H_{0}^{(1)}\left(\sqrt{k_{0}^{2}-\xi^{2}} r\right) \alpha(\xi)
\end{aligned}
$$

здесь $H_{0}^{(1)}$ - функция Ханкеля. После обратного преобразования Фурье находим

$$
v(r, \phi, z)=(2 \pi)^{-3 / 2} \int_{-\infty}^{\infty} \exp (i z \xi) \frac{i}{4} H_{0}^{(1)}\left(\sqrt{k_{0}^{2}-\xi^{2}} r\right) \alpha(\xi) d \xi .
$$

Эта связь дает нам требуемое описание.

Для того чтобы построить самосопряженное расширение, необходимо отобрать из области определения сопряженного оператора множество всех функций, для которых следуюшая "граничная” форма обрашается в нуль:

$$
J(u, v)=\left(A_{L}^{*} u, v\right)-\left(u, A_{L}^{*} v\right)=0, \quad u, v \in D\left(A_{L}^{*}\right) .
$$

В соответствии с (9)

$$
u=u_{0}+u_{L}, \quad u_{0} \in H_{2}=D\left(A_{L}^{\mathrm{F}}\right), \quad u_{L} \in N_{\lambda_{0}} .
$$

Учитьвая (10), получим

$$
J(u, v)=\int_{-\infty}^{\infty}\left(\alpha_{u}^{+}(\xi) \overline{\alpha_{v}^{-}(\xi)}-\alpha_{u}^{-}(\xi) \overline{\alpha_{v}^{+}(\xi)}\right) d \xi .
$$


Здесь $\alpha^{+}$есть $\alpha$ из (10), $\alpha$ принадлежит пространству Соболева $H_{-1}(L)$ (см. [12]), $\alpha_{u}^{-}(\xi)=u_{0}(\xi)$ - след элемента $u_{0}$ из $H_{2}\left(\mathbf{R}^{3}\right)$ на линии $L$. Здесь и ниже мы обозначим через $\alpha(\xi)$ с различными индексами элемент из $H_{-1}(L)$.

Заметим, что область определения сопряженного оператора может быть описана иначе. А именно,

$$
D\left(A_{L}^{*}\right)=D\left(A_{L}\right)+\widetilde{N}_{\lambda_{0}}
$$

где

$$
\widetilde{N}_{\lambda_{0}}=\left\{u: \tilde{u}=\frac{1}{4} \alpha^{+}(\xi) N_{0}\left(\sqrt{k_{0}^{2}-\xi^{2}} r\right)+\frac{i}{4} J_{0}\left(\sqrt{k_{0}^{2}-\xi^{2}} r\right) \alpha^{-}(\xi)\right\},
$$

$J_{0}\left(N_{0}\right)$ есть функция Бесселя (Неймана).

\section{3. ОПИСАНИЕ РАСШИРЕНИЙ}

Мы можем переписать форму $J(u, v)$, используя скалярное произведение в пространстве $H_{-1}(L)$ :

$$
J(u, v)_{-1}=\left(\alpha_{u}^{+}, \alpha_{v}^{-}\right)_{-1}-\left(\alpha_{u}^{-}, \alpha_{v}^{+}\right)_{-1} .
$$

Соотношение $J(u, v)_{-1}=0$ приводит к $J(u, v)=0$. Форма $J(u, v)_{-1}$ есть отображение: $J: H_{-1} \times H_{-1} \rightarrow \mathbf{C}$. Рассмотрим ее действительную и мнимую части:

$$
\begin{aligned}
& \Re J(u, v)_{-1}=\left(\alpha_{u}^{-^{\prime}}, \alpha_{v}^{+^{\prime}}\right)_{-1}+\left(\alpha_{u}^{-^{\prime \prime}}, \alpha_{v}^{+^{\prime \prime}}\right)_{-1}-\left(\alpha_{u}^{+^{\prime}}, \alpha_{v}^{-^{\prime}}\right)_{-1}-\left(\alpha_{u}^{+^{\prime \prime}}, \alpha_{v}^{-^{\prime \prime}}\right)_{-1}, \\
& \Im J(u, v)_{-1}=\left(\alpha_{u}^{-^{\prime \prime}}, \alpha_{v}^{+^{\prime}}\right)_{-1}-\left(\alpha_{u}^{-^{\prime}}, \alpha_{v}^{+^{\prime \prime}}\right)_{-1}+\left(\alpha_{u}^{+^{\prime}}, \alpha_{v}^{-^{\prime \prime}}\right)_{-1}-\left(\alpha_{u}^{+^{\prime \prime}}, \alpha_{v}^{-^{\prime}}\right)_{-1},
\end{aligned}
$$

где

$$
\Re \alpha_{u, v}^{ \pm}=\alpha_{u, v}^{ \pm^{\prime}}, \quad \Im \alpha_{u, v}^{ \pm}=\alpha_{u, v}^{ \pm^{\prime \prime}} .
$$

Сделаем некоторые общие замечания (см. [13]). Пусть $V$ есть сепарабельное гильбертово пространство и $V^{*}$ есть соответствуюшее сопряженное. Любая эрмитова форма $\beta(u, v)$ на $V$ может быть представлена в виде

$$
\beta(u, v)=\sigma(Q u, v)+i \sigma(u, v),
$$

где $Q$ - линейное отображение $(V \rightarrow V)$, которое определяет комплексную структуру, $\sigma(u, v)=\Im \beta(u, v), \sigma: V \times V \rightarrow \mathbf{R}$. Билинейная форма $\sigma$ вырождена и определяет канонический изоморфизм $\widetilde{\sigma}: V \rightarrow V^{*},\langle\widetilde{\sigma} x, y\rangle=\sigma(u, v), x, y \in V$. Пусть $V=L \dot{+} M$, где $L$ и $M$ - трансверсальные лагранжевы плоскости. Поскольку $L=L^{\perp}$ и $M=M^{\perp}$ (относительно $\sigma$ ), дуальность между $L$ и $M$ может быть установлена с использованием $\sigma$ :

$$
\langle x, y\rangle=\sigma(x, y), \quad x \in L, \quad y \in M
$$

$M$ может быть отождествлено с $L^{*}$ и $L-$ с $M^{*}$. Пусть $z=x+y, z^{\prime}=x^{\prime}+y^{\prime}, x, x^{\prime} \in L$, $y, y^{\prime} \in M$. Тогда $\sigma(x, y)=\left\langle x, y^{\prime}\right\rangle-\left\langle x^{\prime}, y\right\rangle$. 
Введем пространство $\left.\widetilde{H_{-1}(\mathbf{R}}\right)=\Re H_{-1}(\mathbf{R}) \dot{+} \Im H_{-1}(\mathbf{R})$. Оно будет совпадать с $H_{-1}(\mathbf{R})$, если ввести комплексную структуру $Q$ в $\widehat{H_{-1}(\mathbf{R})}$. Построим комплексификацию:

$$
H_{-1}^{\mathbf{C}}(\mathbf{R})=\widetilde{H_{-1}(\mathbf{R})}+i \widetilde{H_{-1}(\mathbf{R})}=\left\{\alpha_{u, v}^{+}+i \alpha_{u, v}^{-}, \alpha_{u, v}^{ \pm} \in \widetilde{H_{-1}(\mathbf{R})}\right\} .
$$

Сравнивая (14) с (15), видим, что $\alpha^{+^{\prime}\left({ }^{\prime \prime}\right)}$ принадлежит лагранжевой плоскости и $\alpha^{-^{\prime}\left({ }^{\prime \prime}\right)}$ принадлежит лагранжевой плоскости, которая трансверсальна первой.

Модифицируем результаты из [13]. Введем евклидову структуру на $\widetilde{H_{-1}(\mathbf{R})}$ следующим образом:

$$
\langle\widetilde{u}, \widetilde{v}\rangle=\sigma(T \widetilde{u}, \widetilde{v})=\sigma(\widetilde{u}, T \widetilde{v}) .
$$

Комплексная структура $T$ в $H_{-1}^{\mathbf{C}}(\mathbf{R})$ согласована с $\sigma$. Определение отображения $T$ из леммы 21.6.2 работы [13] имеет вид

$$
\langle u, v\rangle=\sigma(u, T v), \quad \sigma(T u, T v)=0 .
$$

А именно: $\sigma(u, v)=0$, если $u, v$ принадлежат к одной трансверсальной лагранжевой плоскости. Тогда связь $\sigma(u, v)=\sigma(T u, T v)$ приводит к (16). Если $R$ есть другое решение (16), то $\sigma(u, R v)=0$, соответственно $R$ отвечает симметричной форме

$$
A(u, v)=\sigma(R u, T v)=\langle R u, v\rangle,
$$

где $\langle\cdot, \cdot\rangle$ есть комплексная структура, введенная вьше.

Рассмотрим пространство $\widetilde{L_{2}(\mathbf{R})}=\Re L_{2}(\mathbf{R})+\Im L_{2}(\mathbf{R})$, которое переходит в $L_{2}(\mathbf{R})$, если ввести комплексную структуру $Q: Q^{2}=-I$. Форма $A(u, v)$ в $\widetilde{L_{2}(\mathbf{R})}$ есть билинейная симметричная форма, и мы можем представить оператор $R$ как интегральный оператор. Условие $\Im J(u, v)_{-1}=0$ приводит к $\sigma\left(Q \alpha_{+}, \alpha_{-}\right)=\Im J(u, v)_{-1}=0$, где $Q$ есть оператор, определяюший комплексную структуру $L_{2}(\mathbf{R})$. Это означает, что нейтральным подпространством формы $\sigma$ должна быть комплексная плоскость. Тогда нейтральное подпространство формы $J(\cdot, \cdot)$ описывается условием

$$
\alpha_{u}^{+}(z)=\int_{-\infty}^{\infty} B\left(z, z^{\prime}\right) \alpha_{u}^{-}\left(z^{\prime}\right) d z^{\prime},
$$

где $B\left(z, z^{\prime}\right)\left(B\left(z, z^{\prime}\right)=\overline{B\left(z^{\prime}, z\right)}\right)$ есть некоторое ядро.

\section{4. КРУГОВОЙ цИЛИНДР}

Решение задачи рассеяния плоской волны круговым цилиндром (см. [1]) имеет вид

$$
\begin{aligned}
u= & \exp \left(i k_{3} z\right) \sum_{n=-\infty}^{\infty} \exp \left(\operatorname{in}\left(\varphi-\varphi_{0}+\pi / 2\right)\right) \times \\
& \times\left(J_{n}\left(\rho \sqrt{k^{2}-k_{3}^{2}}\right)-c_{n} H_{n}^{(1)}\left(\rho \sqrt{k^{2}-k_{3}^{2}}\right)\right), \\
c_{n}= & \frac{J_{n}\left(d \sqrt{k^{2}-k_{3}^{2}}\right)}{H_{n}^{(1)}\left(d \sqrt{k^{2}-k_{3}^{2}}\right)},
\end{aligned}
$$


здесь

$$
\exp (i(\vec{k}, \vec{x}))=\exp \left(i k_{3} z\right) \sum_{n=-\infty}^{\infty} \exp \left(i n\left(\varphi-\varphi_{0}+\pi / 2\right)\right) J_{n}\left(\rho \sqrt{k^{2}-k_{3}^{2}}\right),
$$

$d$ - радиус цилиндра.

Теперь рассмотрим модель. Принимая во внимание представление (12), (13), можно искать решение задачи рассеяния для модельного оператора в виде

$$
u=\exp \left(i k_{3} z\right)\left(J_{0}\left(\rho \sqrt{k^{2}-k_{3}^{2}}\right)-a \frac{i}{4} H_{0}^{(1)}\left(\rho \sqrt{k^{2}-k_{3}^{2}}\right)\right), \quad a \in \mathbf{C} .
$$

Заметим, что задача сводится к двумерной задаче для случая кругового цилиндра. Следовательно, мы можем выбрать простейший тип расширения для соответствуюшей модели, а именно такой, для которого область определения расширенного оператора описывается условием

$$
\alpha^{+}=\alpha^{-}, \quad \alpha^{-}=\eta \alpha^{+}, \quad \eta \in \mathbf{R}, \quad \alpha^{ \pm} \in \mathbf{C} .
$$

Принимая во внимание представление (12), (13) и асимптотику функций Бесселя, получаем коэффициенты $\alpha^{+}, \alpha^{-}$для $u$. Тогда (20) дает следуюшее выражение для $a$ :

$$
a=\frac{4}{i+i \eta-\frac{2}{\pi} \ln \sqrt{\frac{\lambda^{2}}{\bar{\lambda}_{0}^{2}}}}
$$

где $\lambda=\sqrt{k^{2}-k_{3}^{2}}, \tilde{\lambda}_{0}=\sqrt{k_{0}^{2}-k_{3}^{2}}$.

Сравним полученное модельное решение с соответствующим решением задачи рассеяния для кругового цилиндра радиуса $d$. Видно, что модельное решение совпадает с главным членом (c $n=0)$ ряда (18), если параметры расширения выбраны следуюшим образом:

$$
\eta=0, \quad k_{0}=\frac{2 i}{d} \exp (-\gamma)
$$

$\gamma$ - постоянная Эйлера. Здесь мы учитываем, что $d$ и соответственно $\ln \left(1-k_{3}^{2} / k_{0}^{2}\right)$ являются достаточно малыми.

\section{5. ВОЗМУЩЕННЫЙ ЦИЛИНДР}

Решение модельной задачи рассеяния имеет вид

$$
\begin{aligned}
u= & \exp (i(\vec{k}, \vec{x}))-\exp \left(i k_{3} z\right) \alpha \frac{i}{4} H_{0}^{(1)}\left(\rho \sqrt{k^{2}-k_{3}^{2}}\right)+ \\
& +\frac{i}{4} \int_{-\infty}^{\infty} \exp (i \xi z) \alpha(\xi) H_{0}^{(1)}\left(\rho \sqrt{k^{2}-\xi^{2}}\right) d \xi
\end{aligned}
$$


С другой стороны, элемент из области определения самосопряженного расширения (модельный оператор) представляется в форме (см. вьше)

$$
\begin{gathered}
u=u_{0}+\frac{i}{4} \int_{-\infty}^{\infty} \exp (i \xi z) \alpha^{-}(\xi) J_{0}\left(\rho \sqrt{\widetilde{k}_{0}^{2}-\xi^{2}}\right) d \xi+ \\
+\frac{1}{4} \int_{-\infty}^{\infty} \exp (i \xi z) \alpha^{+}(\xi) N_{0}\left(\rho \sqrt{\widetilde{k}_{0}^{2}-\xi^{2}}\right) d \xi \\
\alpha^{-}(\xi)=\int_{-\infty}^{\infty} B\left(\xi, \xi^{\prime}\right) \alpha^{+}\left(\xi^{\prime}\right) d \xi^{\prime}
\end{gathered}
$$

Мы получаем представление (23) для решения (22) добавлением и вычитанием члена

$$
\frac{1}{4} \exp \left(i k_{3} z\right) \alpha N_{0}\left(\rho \sqrt{k_{0}^{2}-k_{3}^{2}}\right)+\frac{1}{4} \int_{-\infty}^{\infty} \exp (i \xi z) \alpha(\xi) N_{0}\left(\rho \sqrt{\widetilde{k}_{0}^{2}-\xi^{2}}\right) d \xi
$$

Отметим, что $\alpha$ и $\sqrt{k_{0}^{2}-k_{3}^{2}}$ были определены выше (раздел 4). Мы видим, что коэффициенты $\alpha^{ \pm}(\xi)$ для модельного решения (22) следуюшие:

$$
\begin{aligned}
& \alpha^{+}(\xi)=\alpha \delta\left(\xi-k_{3}\right)+\alpha(\xi) \\
& \alpha^{-}(\xi)=\alpha(\xi)\left(-1-\frac{2 i}{\pi} \ln \sqrt{\frac{-\xi^{2}-k^{2}}{-\xi^{2}-\widetilde{k}_{0}^{2}}}\right) .
\end{aligned}
$$

Однако условие в (23) дает нам следующую связь:

$$
\alpha(\xi)\left(-1-\frac{2 i}{\pi} \ln \sqrt{\frac{-\xi^{2}-k^{2}}{-\xi^{2}-\widetilde{k}_{0}^{2}}}\right)=\int_{-\infty}^{\infty} B\left(\xi, \xi^{\prime}\right)\left(\alpha \delta\left(\xi^{\prime}-k_{3}\right)+\alpha\left(\xi^{\prime}\right)\right) d \xi^{\prime} .
$$

Ниже мы сравним полученное модельное решение с соответствуюшим решением задачи рассеяния для тонкого цилиндра.

\section{6. РАССЕЯНИЕ ТОНКИМ ЦИЛИНДРОМ}

В этом разделе мы опишем результаты Федорюка, связанные с рассеянием плоской волны неограниченным осесимметричным цилиндром $S$ малой (в сравнении с длиной волны) толшины $\epsilon$, и сравним их с нашими модельными результатами. Пусть ось цилиндра есть ось цилиндрической системы координат $(r, \theta, z)$ и его форма задана формулой $r=\epsilon \Phi(z)$, где $\Phi$ - гладкая положительно определенная функция. Пусть $u_{0}=$ $\exp (i(\vec{k}, \vec{x}))$ - падающая волна. Рассмотрим уравнение Гельмгольца с граничными условиями Дирихле на цилиндре:

$$
\begin{gathered}
\left(\Delta+k^{2}\right) v(\vec{x})=0, \\
\left.v\right|_{S}=0 .
\end{gathered}
$$

Будем искать решение в форме

$$
v=u+u_{0}+v_{0}
$$


где $v_{0}$ есть рассеянная волна для соответствующего кругового цилиндра. Можно пробовать получить $u$ в форме ряда:

$$
\begin{aligned}
u(\vec{x}) & =\exp \left(i k_{3} z\right) \sum_{n=0}^{\infty} \exp (\operatorname{in} \theta) u_{n}(r, z) \\
\left.u_{n}\right|_{S} & =h_{n}=-J_{n}\left(\sqrt{k^{2}-k_{3}^{2}} r\right)+c_{n} H_{n}^{(1)}\left(\sqrt{k^{2}-k_{3}^{2}} r\right) \\
c_{n} & =\frac{J_{n}\left(\sqrt{k^{2}-k_{3}^{2}} d\right)}{H_{n}^{(1)}\left(\sqrt{k^{2}-k_{3}^{2}} d\right)} .
\end{aligned}
$$

Для тонкого цилиндра толщиной $\epsilon$ решение представимо в форме асимптотического ряда:

$$
u(x)=\sum_{n=0}^{\infty} \epsilon^{n} u_{n}(x) .
$$

Первый член $u_{0}$ этого асимптотического выражения имеет вид

$$
u_{0}(r, z)=\pi(2 i)^{-1} \int_{-\infty}^{\infty} H_{0}^{(1)}\left(\sqrt{k^{2}-\xi^{2}} r\right) \nu_{0}(\xi) \exp (i z \xi) d \xi, \quad \nu_{0}(\xi) \in H_{-1}(L)
$$

где $\nu_{0}(\xi)$ удовлетворяет следуюшему уравнению:

$$
\log \tilde{\epsilon} \sqrt{k^{2}-\xi^{2}} \nu_{0}(\xi)+\int_{-N}^{N} \gamma(\xi-t) \nu_{0}(t) d t=-\tilde{h}(\xi) \eta\left(\frac{\xi}{N}\right) .
$$

Здесь $|\xi|<N=\epsilon^{-1+\delta}, 0<\delta<1, \eta(\xi / N)$ есть характеристическая функция отрезка $[-1,1]$,

$$
\begin{aligned}
& \log \tilde{\epsilon}=\log \epsilon+\frac{\pi}{2 i}-\log 2-\psi(1) \\
& \gamma(\xi)=F(\log \Phi)(\xi), \quad \tilde{h}=F h
\end{aligned}
$$

$F$ - фурье-преобразование, функция $\Phi$ определяет форму цилиндра, функция $h$ есть значение падающей волны на цилиндре.

Функция $h$ в (28) для задачи рассеяния плоской волны имеет вид

$$
c_{0} \int_{-\infty}^{\infty} \delta\left(\xi^{\prime}-k_{3}\right) F(\log \Phi)\left(\xi-\xi^{\prime}\right) d \xi^{\prime}
$$

Выберем функцию $B\left(\xi, \xi^{\prime}\right)=B\left(\xi-\xi^{\prime}\right)=(2 i / \pi) F(\log \Phi)$. Кроме того, пусть модельный параметр $\widetilde{k}_{0}^{2}$ имеет то же значение, что и соответствующий параметр для случая кругового цилиндра, а именно $\widetilde{k}_{0}^{2}=(2 i / d) \exp (-\gamma)$. Тогда для малой толщины $d$ цилиндра и соответственно для большого $\widetilde{k}_{0}^{2}$ имеем

$$
\log \sqrt{\frac{-\xi^{2}-k^{2}}{-\xi^{2}-\widetilde{k}_{0}^{2}}} \cong \log \tilde{\epsilon} \sqrt{-\xi^{2}-k^{2}}
$$

В результате мы получаем совпадение уравнений (25) и (28), т.е. наше модельное решение дает первый член асимптотического ряда (26) для "реального" решения. 


\section{7. СПОНТАННЫЕ РЕШЕНИЯ ДЛЯ КВАЗИГАЗОВОЙ НЕУСТОЙЧИВОЙ СРЕДЫ}

Рассмотрим второе применение оператора Лапласа, возмущенного на множестве нулевой меры, которое связано с квазичаплыгинской средой. Мы можем рассмотреть спонтанные решения рассматриваемой задачи (см. введение), т.е. такие, что возмушения исчезают при $t \rightarrow-\infty$. В этом случае функция $\Phi$ имеет особенность $(\Phi \rightarrow-\infty)$ на окружности $L=\{(r, \phi, z): r=1, z=0\}$. Следовательно, мы должны рассмотреть оператор Лапласа в $L_{2}\left(\mathbf{R}^{3}\right)$, возмушенный на множестве нулевой меры. Это некорректно поставленная задача. Подобные задачи привлекают большое внимание математиков $[6,7,12,14-16]$. Корректный подход основан на теории расширения операторов. Опишем корректную процедуру построения оператора Лапласа в $L_{2}\left(\mathbf{R}^{3}\right)$, возмущенного на окружности (множество коразмерности 2). Мы будем исходить из утверждения, аналогичного утверждению в первой части статьи. Пусть $-\Delta_{0}-$ замкнутый оператор Лапласа, суженный на множество гладких функций, стремящихся к нулю в окрестности $L$.

Лемма $1([14,17])$. Область определения оператора $-\Delta_{0}^{*}$ есть множсество следующих әлементов:

$$
u(\mathbf{x})=\int_{0}^{2 \pi} \frac{\zeta_{u}(\phi) \exp \left(i k_{0}|\mathbf{x}-\mathbf{x}(\phi)|\right)}{4 \pi|\mathbf{x}-\mathbf{x}(\phi)|} d \phi+u_{0}(\mathbf{x}), \quad \mathbf{x}(\phi) \in L
$$

әде $\zeta_{u} \in H_{-1}(L), \quad k_{0}$ есть некоторая комплексная величина $\left(\Im k_{0} \neq 0\right)$.

Лемма 2. Если $u, v \in D\left(-\Delta_{0}^{L *}\right), \quad \zeta_{u}$ есть функиия из представления (29) и $\zeta_{-u}(\phi)=u_{0}(\mathbf{x}(\phi))$, где $u_{0}$ есть функиия из (29), то

$$
-\left(\Delta_{0}^{L *} u, v\right)+\left(u, \Delta_{0}^{L *} v\right)=-\int_{L}\left(\zeta_{u}(\phi) \overline{\zeta_{-v}(\phi)}-\zeta_{-u}(\phi) \overline{\zeta_{v}(\phi)}\right) d l
$$

ДокАЗАТЕЛЬство. Пусть $Z_{\delta}-$ тор, содержащий окружность $L$. Следовательно,

$$
\begin{aligned}
I(u, v) & =-\left(\Delta_{0}^{L *} u, v\right)+\left(u, \Delta_{0}^{L *} v\right)= \\
& =\lim _{\delta \rightarrow 0} \int_{\mathbf{R}^{3}-Z_{\delta}}(-\Delta u \bar{v}+u \overline{\Delta v}) d x .
\end{aligned}
$$

В соответствии с формулой Грина

$$
I(u, v)=\lim _{\delta \rightarrow 0} \int_{\partial Z_{\delta}}\left(\frac{\partial u}{\partial n} \bar{v}-u \overline{\frac{\partial v}{\partial n}}\right) d x
$$

С помощью представления (29) получаем требуемое представление "граничной формы" $I(u, v)$. 
СлЕДСтвиЕ. Область определения симметрического расширения оператора $-\Delta_{0}^{L}$ содержит әлементы и и $v$ из $D\left(\Delta_{0}^{L *}\right)$, для которых

$$
\int_{L}\left(\zeta_{u}(\phi) \overline{\zeta_{-v}(\phi)}-\zeta_{-u}(\phi) \overline{\zeta_{v}(\phi)}\right) d l=0
$$

Ниже мы рассмотрим только наиболее естественные "локальные" расширения, для которых это условие выполняется из-за аннулирования подынтегрального выражения в каждой точке.

Теорема 1. Область определения локального самосопряженного расширения оператора $-\Delta_{0}^{L}$ есть множество всех функиий из области $D\left(\Delta_{0}^{L *}\right)$, которье удовлетворяют “рраничному" условию

$$
\zeta_{-}(\phi)=A(\phi) \zeta(\phi),
$$

где $A(\phi)$ вещественно.

Для того чтобы построить модель с так называемыми высшими моментами, мы должны расширить первоначальное пространство соответствуюшими решениями, не принадлежашими $L_{2}$. Подход аналогичен описанному в работах $[17,18]$. Опишем, как рассмотреть дипольный член. Пусть $\mathcal{A}$ есть следуюшее множество:

$$
\mathcal{A}=\left\{f(\mathbf{x}): f \in L_{2}\left(\mathbf{R}^{3}\right), \int_{\mathbf{R}^{3}} f(\mathbf{x}) \rho^{-2}(\mathbf{x}, L) d V \text { сходится }\right\} .
$$

Здесь $\rho(\mathbf{x}, L)$ - расстояние между $\mathbf{x}$ и $L$. Пусть $h$ - решение, соответствуюшее диполю с осью $l$, проходяшей через точку $\mathbf{x}(\phi)$ кривой $L$ :

$$
h=\frac{\partial}{\partial l} \frac{\exp \left(i k_{0}|\mathbf{x}-\mathbf{x}(\phi)|\right)}{4 \pi|\mathbf{x}-\mathbf{x}(\phi)|},
$$

и

$$
h_{1}=\left(-\Delta-k_{0}^{2}\right)^{-1} h .
$$

Рассмотрим множество $\widetilde{\mathcal{A}}$ :

$$
\widetilde{\mathcal{A}}=\left\{f: f=f_{1}+\int_{0}^{2 \pi} d \phi\left(C_{1}(\phi) h_{1}(\phi)+C(\phi) h(\phi)\right)\right\},
$$

где $f_{1} \in \mathcal{A}$. Мы определим скалярное произведение в $\widetilde{\mathcal{A}}$ в соответствии со следуюшим соотношением:

$$
\begin{aligned}
(f, g)_{\tilde{\mathcal{A}}}= & \left(f_{1}+\int_{0}^{2 \pi} d \phi C_{1}^{f}(\phi) h_{1}(\phi), g_{1}+\int_{0}^{2 \pi} d \phi C_{1}^{g}(\phi) h_{1}(\phi)\right)+ \\
& +\left(f_{1}, \int_{0}^{2 \pi} d \phi C^{g}(\phi) h(\phi)\right)+\left(\int_{0}^{2 \pi} d \phi C^{f}(\phi) h(\phi), g_{1}\right)+ \\
& +\left(\int_{0}^{2 \pi} d \phi C_{1}^{f}(\phi) h_{1}(\phi), \int_{0}^{2 \pi} d \phi C^{g}(\phi) h(\phi)\right)+ \\
& +\left(\int_{0}^{2 \pi} d \phi C^{f}(\phi) h(\phi), \int_{0}^{2 \pi} d \phi C_{1}^{g}(\phi) h_{1}(\phi)\right) .
\end{aligned}
$$


Здесь $(\cdot, \cdot)$ есть $L_{2}$-скалярное произведение. Множество $\widetilde{\mathcal{A}}$ погружено в пространство Крейна обычным образом [19].

Пусть $\widetilde{\Delta}$ - оператор с областью определения

$$
D(\widetilde{\Delta})=\left\{f: f \in \widetilde{\mathcal{A}}, f=f_{2}+\int_{0}^{2 \pi} d \phi C_{1}^{g}(\phi) h_{1}(\phi), f_{2},\left(-\widetilde{\Delta}-k_{0}^{2}\right) f_{2} \in \mathcal{A}\right\} .
$$

На множестве $\mathcal{A}$ оператор $\widetilde{\Delta}$ действует как оператор Лапласа, а на цепочке дополнительных функций оператор $\left(-\widetilde{\Delta}-k_{0}^{2}\right)$ есть оператор сдвига: $\left(-\widetilde{\Delta}-k_{0}^{2}\right) h_{1}=h$. Замкнутый оператор $\widetilde{\Delta}$ является самосопряженным.

Сузим этот оператор на следуюшее множество:

$$
D\left(\Delta_{0}\right)=\left\{f: f \in D(\widetilde{\Delta}),\left(\left(-\widetilde{\Delta}-k_{0}^{2}\right) f, h\right)=0\right\} .
$$

Полученный оператор $\Delta_{0}$ является симметрическим с бесконечными индексами дефекта. Для того чтобы описать его самосопряженные расширения, мы должны рассмотреть "граничную форму"

$$
I(u, v)=-\left(\Delta_{0}^{*} u, v\right)+\left(u, \Delta_{0}^{*} v\right) .
$$

После некоторых вычислений получаем

$$
\int_{L}\left(C_{1 u}(\phi) \overline{C_{v}(\phi)}-C_{u}(\phi) \overline{C_{1 v}(\phi)}\right) d l=0 .
$$

Следовательно, мы приходим к теореме, аналогичной теореме 1.

Теорема 1а. Область определения локального самосопрязсенного расширения оператора $-\Delta_{0}$ есть множество всех функиий из области $D\left(\Delta_{0}^{*}\right)$, которые удовлетворяют "граничному" условию

$$
C_{1}(\phi)=B(\phi) C(\phi)
$$

где $B(\phi)$ вещественно.

Рассмотрим нашу задачу. Мы можем представить решение уравнения Лапласа с "граничным" условием на окружности, используя тороидальные координаты $(\sigma, \phi, \eta)$ :

$$
r=(\operatorname{ch} \sigma+\cos \eta)^{-1} \operatorname{sh} \sigma, \quad z=(\operatorname{ch} \sigma+\cos \eta)^{-1} \sin \eta .
$$

Тогда уравнение Лапласа принимает вид

$$
\left(r \Phi_{\sigma}^{\prime}\right)_{\sigma}^{\prime}+\left(r \Phi_{\eta}^{\prime}\right)_{\eta}^{\prime}+(\operatorname{ch} \sigma+\cos \eta)^{-2} r^{-1} \Phi_{\phi \phi}^{\prime \prime}=0 .
$$

Пусть

$$
\Phi=r^{-1 / 2} f \cos (m \phi), \quad f=\sum_{n=0}^{\infty} f_{n}(\sigma) \cos \left(n \eta+\eta_{n}\right)
$$

5 Теоретическая и математическая физика, т. 119, № 2, 1999 г. 
Следовательно, функции $f_{n}$ удовлетворяют уравнению Лежандра

$$
\left(\left(1-y^{2}\right) f_{y}^{\prime}\right)_{y}^{\prime}+\left(\nu(\nu+1)-n^{2}\left(1-y^{2}\right)^{-1}\right) f=0
$$

где $y=\operatorname{cth} \sigma, \nu=-m-1 / 2$. Решениями (33) являются функции Лежандра $P_{\nu}^{n}$ и $Q_{\nu}^{n}$. Только функции Лежандра второго рода $Q_{\nu}^{n}$ связаны со спонтанными решениями, т.к. они имеют особенность при $y=1$ (т.е. на окружности $r=1$ ). В $L_{2}\left(\mathbf{R}^{3}\right)$ имеется одно фундаментальное решение $r^{\nu} Q_{\nu}$. Если мы хотим рассмотреть слагаемые дипольного типа, т.е. $r^{\nu} Q_{\nu}^{1} \cos \eta, r^{\nu} Q_{\nu}^{1} \sin \eta$, необходимо построить соответствуюший оператор в расширенном пространстве (пространстве Крейна). Если рассматривается только первый член, то полное решение есть интеграл от $r^{\nu} Q_{\nu}$ по окружности с некоторым весом $w(\phi)$. Для обеспечения знака особенности необходимо выбрать знак $w(w<0)$. Но для дипольного члена ситуация иная. Это фундаментальное решение имеет разные знаки для различных значений $\eta(-\pi / 2<\eta<\pi / 2$ и $\pi / 2<\eta<3 \pi / 2)$. Некоторые авторы [11] преодолевают это препятствие выбором параметров с различными знаками для "левой” и “правой” частей диполя. Конечно, очень странно иметь диполь с двумя "минусами". Тем не менее такое решение используют, обрашая внимание на тот факт, что в этом случае $\Phi$ - не электростатический потенциал, а некоторая формальная функция. Но есть другой (математический) аргумент: производная функции $\Phi$ в этом случае не является непрерывной. Этот факт не обсуждается в [11].

Рассмотрим “корректный” диполь. Тогда для некоторых значений $\eta$ мы имеем $\Phi \rightarrow$ $\infty$ на окружности, а для некоторых значений $\Phi \rightarrow-\infty$ на окружности. Это означает, что существуют “спонтанные" (растущие) и “убывающие” возмущения для различных значений $\rho, v$. Одна область соответствует нестабильности относительно возмущений такого типа, другая область соответствует стабильности. Границей является следуюшая поверхность в нашем “формальном” пространстве: $\{(\sigma, \phi, \eta): \eta= \pm \pi / 2\}$, т.е. $\left\{(r, \phi, z): r^{2}+z^{2}=1\right\}$. Эта поверхность соответствует следуюшей связи между плотностью и скоростью:

$$
\rho_{*}^{1 / m}+\frac{v^{2}}{\left(2 m c_{0}\right)^{2}}=1 .
$$

Это соотношение дает границу области стабильности/нестабильности на $\rho, v$-плоскости.

ЗАмЕчАниЕ. Мы рассматривали здесь только локальные расширения. Эти расширения соответствуют спонтанным решениям, описанным в [11]. Но существуют нелокальные расширения, построение которых аналогично проведенному в первой части статьи. Эти расширения могут соответствовать другим физическим спонтанным решениям, не описанным в физической литературе.

Благодарности. Работа частично поддержана фондом Сороса. 


\section{Список литературы}

[1] М. В. Федорюк. Изв. АН СССР. Сер. матем. 1985. Т. 49. № 1. С. 160-193.

[2] М. В. Федорюк. Изв. АН СССР. Сер. матем. 1981. Т. 45. № 1. С. 165-186.

[3] В. М. Бабич, Б. А. Самокии, Д. Б. Дементьев. Зап. научн. сем. ПОМИ РАН. 1995. T. 221. C. $67-74$.

[4] E. Fermi. Ric. Sci. 1936. V. 7. P. 13-52.

[5] Ф. А. Березин, Л. Д. Фаддеев. ДАН СССР. 1961. Т. 137. № 5. С. 1011-1014.

[6] S. Albeverio, F. Gesztesy, R. Hoegh-Krohn, H. Holden. Solvable Models in Quantum Mechanics. Berlin: Springer, 1988.

[7] Б. С. Павлов. УМН. 1987. Т. 42. № 6. С. 99-131.

[8] D. E. Potter. Nuclear Fusion. 1978. V. 18. P. 813-820.

[9] A. Shyam, M. Srinivasan. Appl. Phys. 1978. V. 17. P. 245-252.

[10] Я. Б. Зельдович, И. Д. Новиков. Структура и эволюция Вселенной. М.: Наука, 1975.

[11] С. К. ЖҚанов, Б. А. Трубников. Квазигазовые неустойчивые среды. М.: Наука, 1991.

[12] А. С. Благовещенский, К. К. Лаврентьев. Вестн. Ленингр. унив. 1977. № 1. С. 9-16.

[13] L. Hormander. The Analysis of Linear Partial Differential Operators. 3. Pseudo-Differential Operators. Berlin-Heidelberg-NewYork-Tokyo: Springer-Verlag, 1985.

[14] Ю. Г. Шондин. ТМФ. 1995. Т. 105. С. 3-17.

[15] С. П. Новиков, Б. Ю. Стернин. ДАН СССР. 1966. Т. 170. С. 1265-1267.

[16] V. A. Geyler, B. S. Pavlov, I. Yu. Popov. J. Math. Phys. 1996. V. 37. P. 5171-5194.

[17] I. Yu. Popov. J. Math. Phys. 1992. V. 33. № 11. P. 3794-3801.

[18] Ю. Г. Шондин. ТМФ. 1988. Т. 74. С. 331-344.

[19] J. Bognar. Indefinite Inner Product Spaces. Berlin: Springer, 1975.

Поступила в редакцию 2.IX.1998 г., после доработки 2.XI.1998 г. 